\title{
Microstructural Evolution during Heat Treatment of 3D Printed Maraging Steel
}

\author{
Francis Tetteh ${ }^{*}$ and Solomon Boakye-Yiadom
}

Mechanical Engineering Department, York University, Toronto, ON, Canada.

*Corresponding author: francistetteh20@yahoo.com

Maraging steels are advance high strength steels which have gained much interest in defense, aerospace, energy and automotive industries where high strength and fracture toughness is required [1,2]. These type of steels are special type of low carbon steels known for their ultrahigh strength, high fracture toughness, good weldability and machinability [3,4]. The low carbon contents of this steel makes them less susceptible to quench cracking and corrosion that may result in failure due to the absence of carbide precipitations [4]. However, age- hardening treatment is used to achieve precipitation of intermetallic compounds that enhances its mechanical properties since the initial microstructure comprises mainly of soft martensitic structures from its low carbon content [2].

The recent advances in additive manufacturing (AM) or Metal 3D Printing has proven to be a revolutionary manufacturing technique for fabricating parts with complex geometry from 3D drawing of the part [1]. This field is gaining interest in the tooling, medical and aerospace industries for rapid prototyping. They offer design flexibility to produce intricate shapes for tooling applications, customized medical parts, complex porous and lattice structures by simply making computer drawings of components $[5,6]$. One of the most important Metal 3D printing technique is selective laser sintering where metallic powders are successively consolidated layer by layer on top of each other using laser as the source of energy $[1,4]$. Most recently, the SLS technique was adopted for printing maraging steel and evaluating the microstructural integrity of the as-printed material. Due to the presence of non-equilibrium phases and residual stresses, it is recommended that heat treatment be used to improve the structure and properties.

In this study, 3D printed maraging steel were heat-treated at different temperatures and time intervals to evaluate the effect of heat treatment on the microstructure and properties of the specimens. Microstructural characterization techniques including the use of Transmission Electron Microscopy (TEM) and X-Ray diffraction (XRD) were used to characterize the resulting microstructures of both the as-printed and heattreated specimens to study how heat treatment affects the microstructural evolution of the specimens.

TEM analysis of the as-fabricated material revealed martensitic structures within the microstructure without any observable second phase particles as shown in Fig. 1(a). The structure was engulfed within buildup of high dislocation cell structures. Also, the extent of precipitation was minimal or limited. However, the quantity of precipitates increased and formed large spherical carbides at increasing heat treatment durations as shown in Fig. 1 (b and c) at 4hrs and 8hrs respectively. EDX analysis of the elemental composition shown in Fig. 2 gave a chemistry of Ni, Al, Fe, Mo, Ti, and Co. The second phase was a reverse austenite but mostly the matrix fully composed of the martensitic lath structures within cubic iron. The different phases present in the maraging steel in the as-fabricated and at increasing heat treatment times is shown in the XRD patterns of Fig. 3. XRD patterns of all samples showed 2 characteristic peaks of martensitic (a) phases within cubic iron occurring at atomic planes of (110),) and (200) respectively. The patterns revealed a gradual decrease in peak intensities with a continues broadening of the peaks at increasing heat treatment times. In addition, at increasing heat treatment times, there was a progressive 
increase in volume fraction of a second phase (austenite (y)) identified at atomic plane of (111) as observed from the XRD patterns in Fig. 1(b-c). The broadening and reduction of the peaks at increasing heat treatment times maybe attributed to increasing amount of precipitation and reversion of austenitic phase.

Fabrication of 3D printed maraging steel leads to the formation of martensitic lath structures within cubic iron with high volume of dislocation cell structures with minimum precipitates. In order to optimize the structural integrity such as strength of the as printed material, heat treatment must be done to increase particle precipitation and second phases to enhance the materials properties.

\section{References:}

[1] C. Tan et al., Materials and Design 134(2017) p.13.

[2] K. Kempen et al., Physics Procedia 12 (2011) p. 255.

[3] G.K. Singh, M.M. Ghosh, Transactions of the Indian Institute of Metals 70(8) (2017). p. 2111.

[4] A. Alafaghani, A. Qattawi, M.A.G Castañón, The International Journal of Advance Manufacturing Technology (2018) p. 2491.

[5] C.L. Tan, et al., Proceedings of the 2016 6th International Conference on Advanced Design and Manufacturing Engineering 96(2016) p. 404.

[6] E.A. Jägle, et al., Journal of Materials Research, 29 (2014) p.2072.

[7] I acknowledge my supervisor for his contributions towards this work.
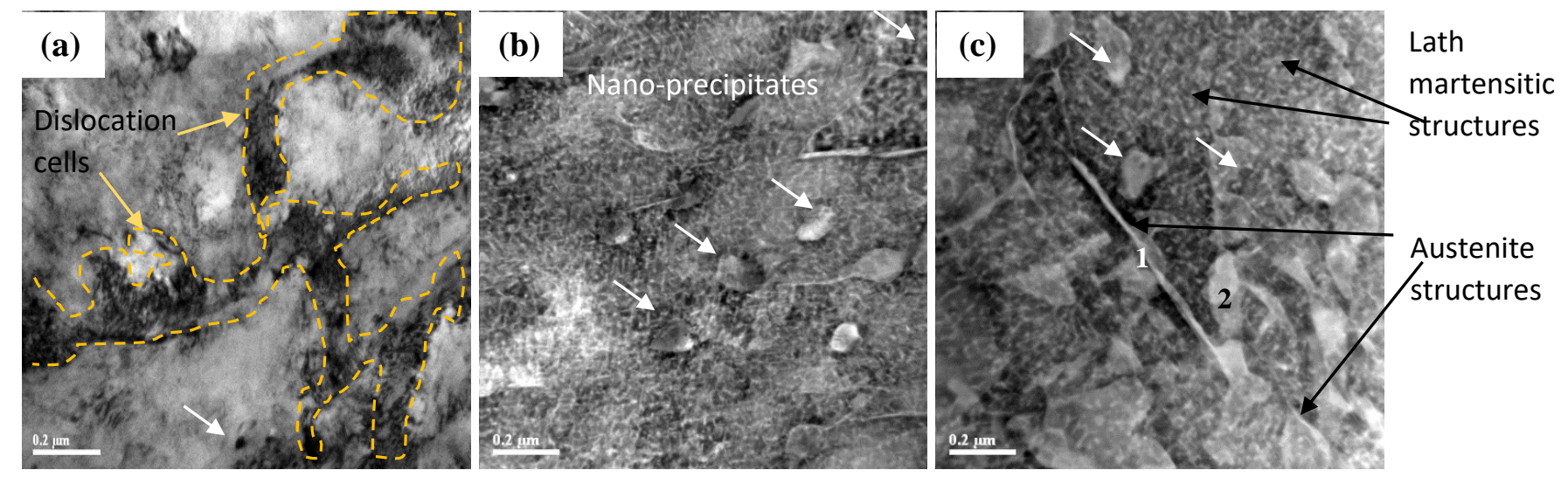

Figure 1. TEM micrographs of a) initial as fabricated structure b) aged heat treated at 4 hrs. c) at $8 \mathrm{hrs}$
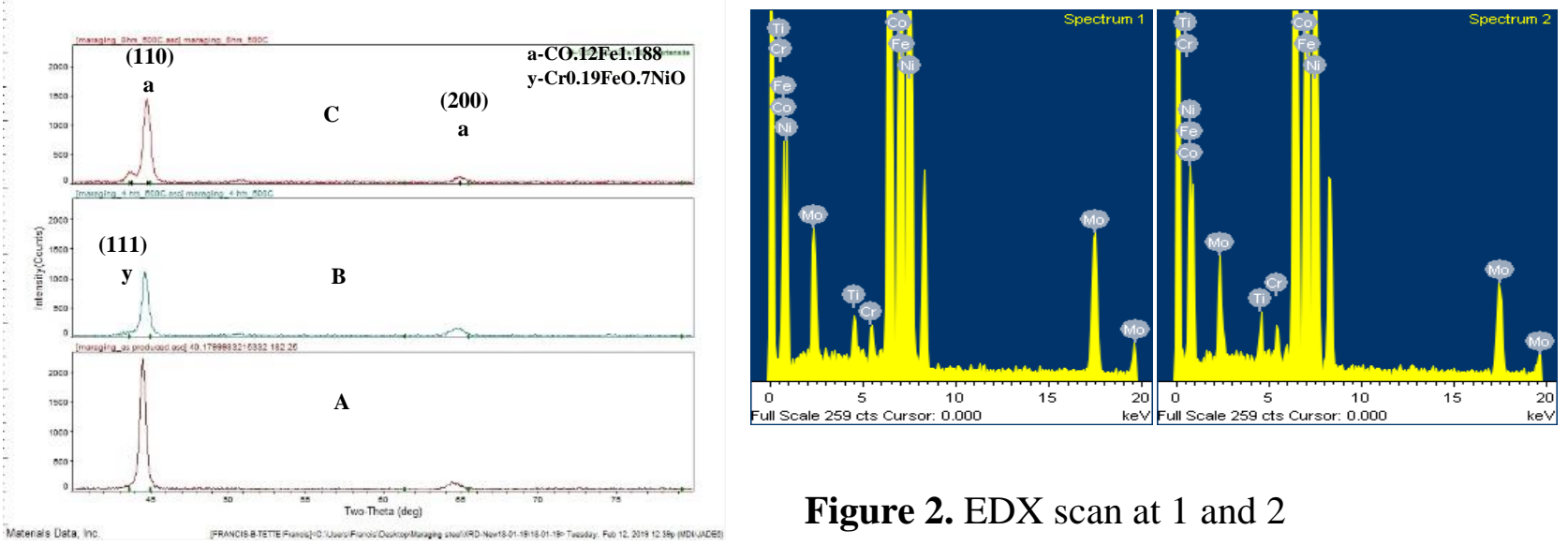

Figure 2. EDX scan at 1 and 2

Figure 3. XRD patterns of a) as fabricated, $500{ }^{\circ} \mathrm{C}$ aged b) 4 and c) 8 hours 